\title{
Il y a toujours un chameau qui tombe. Les multiples fonctions des clivées en il y a
}

\author{
Lena Karssenberg $^{1^{*}}$ \\ ${ }^{1}$ KU Leuven, Blijde-Inkomststraat 21, 3000 Leuven, Belgique
}

Résumé. Dans cet article, je me propose d'étudier les fonctions des clivées en il y a ('Il y a le chat qui miaule') en contraste avec leurs équivalents canoniques à l'ordre des mots Sujet Verbe ('Le chat miaule'). Contrairement à ce que laissent penser certaines études antérieures, je montrerai que ces clivées ne sont pas uniquement motivées par la focalisation / le marquage de la structure informationnelle. Je présenterai des exemples de corpus de clivées en il y a qui ont des fonctions diverses telles que l'expression de la négation emphatique et le renforcement de la cohérence textuelle, que leurs équivalents canoniques n'ont pas. Autrement dit, quand le locuteur utilise une clivée en il y a plutôt que son équivalent à l'ordre des mots Sujet Verbe, ce choix ne s'explique pas uniquement par la fonction de la focalisation. Ainsi, cette analyse s'inscrit dans une tendance plus générale: des unités linguistiques qui étaient auparavant considérées comme de simples marqueurs de focus s'avèrent de plus en plus souvent multifonctionnelles.

\begin{abstract}
There's always a camel that falls. The multiple functions of il $y$ a ('there is') clefts. In this article, I investigate the functions of French il y a clefts such as Il y a le chat qui miaule ('The cat is meowing', literally 'There is the cat that is meowing') in contrast with their canonical equivalents with Subject Verb word order (Le chat miaule 'The cat is meowing'). Contrary to previous assumptions, I show that such clefts are not solely motivated by information structure. I will present corpus examples of il y $a$ clefts that have various functions such as the expression of reinforced negation and the enhancement of textual coherence, which their canonical counterparts do not have. Therefore, when a speaker uses an il y a cleft rather than its Subject Verb counterpart, this choice cannot be explained by referring to the focus marking function alone. In this sense, my analysis is in line with a more general tendency: certain linguistic units that used to be considered as simple focus markers turn out to be multifunctional.
\end{abstract}

* Corresponding author : lena.karssenberg@kuleuven.be 


\section{Introduction}

Les clivées en c'est sont très souvent considérées comme des constructions dont la raison d'être est d'exprimer une certaine structure informationnelle qui n'est généralement pas associée aux phrases à l'ordre des mots Sujet Verbe (SV). Considérons par exemple la clivée en (1a) et son équivalent canonique à l'ordre des mots SV en (1b).

\section{- Qui a mangé le fromage? \\ a. C'est le chat qui l'a mangé. \\ b. Le chat l'a mangé.}

La clivée en (1a) permet de focaliser l'élément clivé le chat, alors que la relative exprime l'arrière-fond (voir par exemple Smits, 1989 ; Clech-Darbon et al., 1999 ; Katz, 2000 ; Lambrecht, 2001 ; Rialland et al., 2002 ; Doetjes et al., 2004 ; Dufter, 2008 ; Mertens, 2008 ; Destruel, 2013 ; Lahousse \& Borremans, 2014). Les phrases canoniques telles que (1b), par contre, ne sont généralement pas associées à l'interprétation informationnelle 'focus + arrière-fond'. Par conséquent, le locuteur choisira la version clivée plutôt que son équivalent à l'ordre des mots SV s'il veut focaliser le chat (Lambrecht, 1994).

Cependant, l'idée que les clivées en c'est (et leurs équivalents à travers les langues) soient de simples marqueurs de focus a reçu de plus en plus de critiques. Premièrement, toutes les clivées en c'est n'ont pas l'interprétation 'focus + arrière-fond' ; l'interprétation 'topique + commentaire' est également possible (voir Doetjes et al., 2004 ; BlancheBenveniste, 2006 ; Dufter, 2008, 2009 ; Mertens, 2012 ; Lahousse \& Borremans, 2014). Deuxièmement, les clivées en c'est et en it peuvent également avoir d'autres motivations qui ne sont pas directement liées aux notions de 'topique' et de 'focus'. Ainsi, Dufter (2009) montre que l'emploi d'une clivée en c'est peut permettre d'éviter des ambiguïtés de portée et renforcer la cohérence textuelle. Les mêmes fonctions ont été attribuées aux clivées en it par Delin (1989) et Delin \& Oberlander (1995). Cela amène Delin \& Oberlander (1995) à la conclusion suivante : «(..) we take clefts, like many other pragmatically marked syntactic constructions, to convey not a single semantic or pragmatic feature but a bundle of such features, acting jointly to determine the discourse functions a sentence type can serve. [...] it seems plausible to assume that speakers choose a sentence type that best fits their conversational goals in a given context, and that a range of factors, rather than a single one, determines this choice " (Delin \& Oberlander, 1995: 470, mes italiques). Dans le même ordre d'idées, Dufter (2009 : 108) affirme : "By all odds, there must be more to clefting than signaling focus $»$.

Le but de cet article est de présenter des preuves supplémentaires pour montrer que les clivées sont plus que des marqueurs de focus. Pour ce faire, j'analyserai un type de clivée française moins connue que la clivée en c'est, à savoir la clivée en il y a. Dans la plupart des cas, ce type de clivée a une interprétation 'thétique' ou 'tout-focus' où toute l'information est nouvelle :

$$
\begin{aligned}
& \text {-Qu'est-ce qui se passe? } \\
& \text { - Il y a le chat qui miaule. }
\end{aligned}
$$

Plusieurs auteurs affirment que les francophones utilisent des clivées en il y a comme (2) pour introduire un nouveau référent qui est focal (et non topical) (par exemple Lambrecht, 1988, 1994 ; Karssenberg, 2016a). Autrement dit, la motivation de telles clivées résiderait dans la structure informationnelle. Je ne contesterai pas que les clivées en il y a peuvent être utilisées pour marquer des interprétations informationnelles particulières (voir Lambrecht, 1986, 1988, 1994 ; Karssenberg, 2016a, 2016b). Cependant, je montrerai à l'aide de données de corpus que les fonctions des clivées en il y a ne se réduisent pas au 
marquage informationnel. Dans ce sens, mon analyse est en accord avec une tendance plus générale dans le domaine de la structure informationnelle : il s'est avéré que plusieurs structures (parmi lesquelles les clivées en c'est, voir ci-dessus) qui étaient auparavant vues comme des marqueurs de focus sont en réalité multifonctionnelles (Matić \& Wedgwood, 2012).

La structure de cet article est la suivante : je ferai d'abord un survol des analyses antérieures des clivées en il y a (section 2). Dans la section 3, je présenterai les corpus utilisés. Suivent alors les trois « nouvelles » fonctions des clivées en il y $a$ : le renforcement de la cohérence textuelle (section 4), la négation emphatique (section 5) et l'interprétation «plurielle » des SN indéfinis singuliers (section 6).

\title{
2 Analyses antérieures
}

Dans ce qui suit, je présenterai les propriétés les plus importantes des clivées en il y a (section 2.1) et leurs fonctions (section 2.2), telles qu'elles ont été présentées dans les principales analyses antérieures.

\subsection{Les propriétés principales des clivées en il y a}

Les clivées en il y a sont surtout attestées à l'oral (Lambrecht, 1986 ; Blanche-Benveniste, 1997 ; Katz, 2000 ; Choi-Jonin \& Lagae, 2005 ; Dufter, 2008 ; Willems \& Meulleman, 2010 ; Karssenberg, 2016a). Plusieurs propriétés distinguent ces clivées d'autres phrases en il y a, non-clivées (voir notamment Léard, 1992 ; Lagae \& Rouget, 1998 ; Choi-Jonin \& Lagae, 2005 ; Verwimp \& Lahousse, 2016 ; Karssenberg, 2017b pour plus de détails concernant les tests et propriétés qui distinguent les clivées en il y a des autres types de phrase). Une distinction est souvent présentée entre (i) les clivées en il y a ayant une interprétation événementielle (3), (ii) les phrases en il y a existentielles (4) et (iii) les phrases en il y a locatives (5).

(3) Clivée en il y a (interprétation événementielle)

Y'a Jean qui s'est cassé la jambe. (Lambrecht, 1986 : 115)

(4) Phrase en il y a existentielle

Mais il y a des gens qui sont allergiques aux piqûres d'abeilles.

(Choi-Jonin \& Lagae, $2005: 43$ )

\author{
Phrase en il y a locative \\ Sur l'autre trottoir, il y a quelques hommes qui sont gros. \\ (Choi-Jonin \& Lagae, $2005: 45$ )
}

Seules les clivées correspondent à un équivalent à l'ordre des mots SV (6), et contrairement aux phrases existentielles et locatives, la relative dans les clivées en il y a est prédicative plutôt que restrictive ou appositive (Lagae \& Rouget, 1998 ; Choi-Jonin \& Lagae, 2005 ; Karssenberg, 2017b, inter alia).

(6) Équivalent canonique à l'ordre des mots SV

Jean s'est cassé la jambe. (Lambrecht, $1986: 115$ ) 
Notez que toutes les clivées en il y a ne sont pas événementielles : elles peuvent également être spécificationnelles (7) (voir entre autres Lambrecht, 2001 ; Verwimp \& Lahousse, 2016 ; Karssenberg, 2017a). Dans ce cas-là, la clivée présente une valeur (celui d'la Roquette) pour une variable (espaces verts).

\author{
Clivée en il y a (interprétation spécificationnelle) \\ - A : et alors vous manquez un peu d'espaces verts tu dis vers le square \\ - B : on a un petit square \\ - C : euh oui oui y a celui-là y a celui d'la Roquette qu'est pas loin (...)
}

(Verwimp \& Lahousse, 2016 : 13)

\title{
2.2 Les fonctions des clivées en il y a
}

Comme mentionné dans l'introduction, la plupart des auteurs qui analysent les clivées en il y a estiment que la motivation de ces clivées réside dans le domaine de la structure informationnelle. Les clivées en il y a sont souvent appelées "présentationnelles », parce que l'une de leurs fonctions principales serait d'introduire un nouveau référent ou événement dans le discours (Lambrecht, 1986 ; Giry-Schneider, 1988 ; Lambrecht, 1988, 1994 ; Ashby, 1995, 1999 ; Lambrecht, 2000a, 2000b, 2001, 2002 ; Jullien, 2007 ; Pitavy, 2009 ; Secova, 2010). Plus spécifiquement, selon Lambrecht (1988, 1994), la structure clivée signale au locuteur que la phrase n'est pas à interpréter comme une phrase en 'topique + commentaire', contrairement aux phrases canoniques à l'ordre des mots SV. Ainsi, dans un contexte où Jean n'a pas encore été mentionné, la clivée en (8a) est beaucoup plus naturelle que son équivalent canonique en ( $8 \mathrm{~b})$ (surtout à l'oral), parce qu'en français, l'ordre des mots SV est généralement associé à l'interprétation 'topique + commentaire' plutôt qu'à l'interprétation 'tout-focus'.
a. Y'a Jean qui s'est cassé la jambe.
b. Jean s'est cassé la jambe.
(Lambrecht, 1986 : 115)

Karssenberg (2016a) tire une conclusion similaire, en précisant toutefois que ce n'est pas la structure clivée qui a cette fonction, mais uniquement l'expression il y a, parce que les phrases existentielles en il y a sans relative n'ont pas l'interprétation 'topique + commentaire' non plus. Notez, cependant, que toutes les clivées en il y a n'ont pas l'interprétation 'tout-focus'; elles peuvent également avoir les articulations 'focus + arrière-fond' (9) (Lambrecht, 1988, 2001 ; Verwimp \& Lahousse, 2016) ainsi que 'topique contrastif + commentaire' (10) (Karssenberg, 2016b, 2017b ; Karssenberg \& Lahousse, à venir). ${ }^{\mathrm{i}}$

\author{
Focus + arrière-fond \\ Y'a Beth qui veut y aller, euh, y'a y'a Jean-Marc, y'a moi, bon. \\ (Lambrecht $1988: 154$ )
}

\author{
Topique contrastif + commentaire \\ " Nous, on a de la chance, on a des employeurs. Mais il y a plein de petites jeunes \\ qui se font avoir », poursuit Lina.
}

(Karssenberg $2017: 193$ )

Un nombre limité d'études mentionnent des fonctions des clivées en il y a qui ne sont pas directement liées aux notions de 'topique' et de 'focus'. Ainsi, Jullien (2007) et Pekarek 
Doehler (2015) présentent des analyses interactionnelles des clivées en il y a et affirment que le segment 'il y a SN' est une "Turn Constructional Unit» qui projette une continuation sous forme de relative prédicative ou autre. L'emploi de la clivée en il y a permet donc au locuteur de gérer la manière dont se déroule son tour de parole, selon ces auteurs. De plus, Karssenberg (2017a) montre que les relatives dans les clivées en il y a du type 'focus + arrière-fond' peuvent permettre au locuteur de signaler que la proposition exprimée par la clivée est pertinente par rapport à la «Question Under Discussion » et que ces relatives peuvent également exprimer un jugement de la part de l'interlocuteur. La fonction de la 'pertinence' est illustrée par (11) : en répétant la variable (moins de 10.000 euros), le locuteur $\mathrm{B}$ souligne qu'il/elle répond bel et bien à la question posée par le locuteur A. D'après Karssenberg (2017a), cela expliquerai au moins en partie pourquoi le locuteur opte pour une structure clivée plutôt que pour une phrase existentielle sans relative ('Il y a la Citroën C1. '), qui serait également possible dans ce contexte.

\section{Clivée en il y a spécificationnelle : relative qui signale la pertinence \\ - A : Je recherche des modèles de voiture à acheter neuve moins de 10 000 euros, ou aller!? \\ - B : $\quad$ bonjours. il y a la citroen c1 qui est a moins de 10000 euros.}

(Karssenberg, 2017a: 82-83, exemple de corpus - erreurs authentiques non corrigées)

\section{Corpus et extraction}

Les deux corpus utilisés pour cette étude sont YCCQA (Yahoo-based Contrastive Corpus of Questions and Answers) ${ }^{\mathrm{ii}}$ et Le Monde (1998). Le corpus YCCQA contient environ 6,1 million de mots et est basé sur le forum de discussion Yahoo Questions et Réponses. Ce forum est une plateforme où l'on peut poser des questions sur des sujets très divers (la religion, les animaux, la politique, les jeux vidéo, et cetera) (voir aussi Lahousse \& Lamiroy, 2015). Le corpus contient énormément d'erreurs d'orthographe, que je n'ai pas corrigées dans les exemples que je présente ci-dessous. Le corpus Le Monde contient toutes les éditions du Monde parues en 1998 (voir Verlinde \& Selva, 2001b, 2001a).

Toutes les phrases en il y a... qui/que ont été extraites des deux corpus. Pour la classification des données de corpus, j'ai tenu compte des deux critères principaux présentés dans la section 2.1 : la possibilité de « décliver» la phrase (c'est-àdire la transformer en une phrase à l'ordre des mots SV) et le type de relative (ni restrictive, ni appositive). Au total, 366 phrases en il y a ... qui/que correspondent à ces critères (285 clivées en il y a dans YCCQA et 81 clivées en il y a dans Le Monde).

\section{La cohérence textuelle}

Dans la littérature sur les clivées en c'est et en it, il a été remarqué que ces clivées peuvent servir à renforcer la cohérence textuelle (voir par exemple Krötsch \& Sabban, 1990 ; Dufter, 2009 ; Lahousse \& Borremans, 2014 ; Jacob, 2015 sur les clivées en c'est ; Delin, 1989 sur les clivées en it). Ceci est particulièrement clair quand l'élément clivé est anaphorique, comme en (12). En mettant l'expression anaphorique devant la prédication dans la relative, le lien avec ce qui précède est plus clair ; on passe du connu au nouveau. ${ }^{\text {ii }}$

\footnotetext{
Monsieur le Commissaire, lisez l'avis de la commission de l'emploi et des affaires sociales et accordez-lui un caractère prioritaire, car c'est là que réside le principal problème des citoyens.

(Jacob, $2015: 111$ )
} 
Le même raisonnement vaut pour les clivées en il y a qui contiennent des expressions anaphoriques telles que comme cela en (13). Le lien entre l'anaphore et le contexte précédant est plus clair dans la clivée qu'en (14), où comme cela est en position finale et donc plus éloigné de son antécédent dans la phrase précédente.

- A : Faire des concessions signifie-t-il être une bonne poire?

- B : Non! Dans une vie de couple, il faut savoir faire des concessions. Cela ne veut pas dire laisser ses envies et ses opinions de côté. Les 2 partenaires doivent faire des concessions, il n'y a que comme cela que le couple peut durer! (YCCQA)

(14) Les deux partenaires doivent faire des concessions, le couple peut seulement durer comme cela!

Il est vrai que le syntagme adverbial pourrait également être placé en tête de phrase (seulement comme ça, le couple...). Cependant, dans cette position, les adverbes de manière ont tendance à être interprétées comme des adverbes extraprédicatifs (Molinier \& Levrier, 2000 inter alia $^{\text {iv }}$, cf. Lamiroy \& Charolles, 2004 sur les interprétations et la position de seulement). Étant donné que comme ça exprime une manière en (13) (c'est-àdire une interprétation intraprédicative), il ne serait pas opportun de mettre comme ça en tête de phrase, une position normalement associée aux adverbiaux à interprétation extraprédicative (par exemple la conséquence au lieu de la manière).

Il est intéressant de noter qu'un certain nombre de relatives dans les clivées en il y a avec des expressions anaphoriques sont assez longues. Si l'on transforme de telles clivées en des phrases à l'ordre des mots SV, l'expression anaphorique, qui est un syntagme assez court, suit une suite de mots plus longue. Ceci va à l'encontre de la préférence générale « syntagme court - syntagme long» («the Principle of End Weight»: Behagel, 1909 ; Quirk et al., 1985 ; Hawkins, 1994 ; Wasow, 2002). Considérons par exemple (15). L'élément clivé en Turquie est donné en discours : l'article dans Le Monde d'où provient cet extrait porte sur la Turquie, qui est évoquée par son pays et $y$ dans la phrase précédant la clivée. La relative exprime des informations nouvelles et elle est très longue. Par conséquent, il ne serait pas naturel de placer seulement en Turquie à la fin de la phrase, comme en (16).

(15) Mais l'essentiel de la marque qu'Atatürk a imposée à son pays semble indélébile. Personne d'ailleurs n'y parle sérieusement de remettre son ouvre en question. Et $\underline{i l}$ n'y a qu'en Turquie que le dirigeant du parti intégriste a pu faire alliance, sans que personne parmi ses partisans s'en choque, avec une femme aussi visiblement occidentalisée que Tansu Ciller, économiste made in USA. (Le Monde)

(16) \# Et le dirigeant du parti intégriste a pu faire alliance, sans que personne parmi ses partisans s'en choque, avec une femme aussi visiblement occidentalisée que Tansu Çiller, économiste made in USA seulement en Turquie.

De nouveau, il est possible de placer seulement en Turquie en tête de phrase, mais cette position est de préférence réservée aux adverbiaux de phrase plutôt qu'aux adverbes à interprétation de manière (Molinier \& Levrier, 2000 ; Lamiroy \& Charolles, 2004). Autrement dit, la longueur des syntagmes peut être considérée comme une raison supplémentaire pour la préférence du format clivé plutôt que l'ordre des mots SV.

Notez également que dans certains cas, une clivée en il n'y a que a une interprétation de négation exceptive et correspond alors à un équivalent canonique où la phrase 
commence par 'seul N'. Cependant, cette transformation entraîne un changement de registre involontaire. Ainsi, la clivée en (17) correspond à l'équivalent canonique en (18), mais les phrases où «seul(e)(s) X» se trouve en position de sujet préverbal sont généralement perçues comme formelles (Lamiroy \& De Mulder, 2011 : 309). Par conséquent, la phrase en (18) ne conviendrait pas aussi bien que la clivée en (17) dans le contexte original : cet extrait vient du forum en ligne Yahoo Questions et Réponses, qui est caractérisé par un style de français informel.

\section{Clivée en il n'y a que}

- A : Comment faire pour effacer de ma tête l'image du cadavre que j'ai retrouvé hier dans un ravin?

- B : je crois qu'il n'y a que le temps qui te fera oublier cette image. (YCCQA)

\section{Équivalent canonique en 'seul $\mathbf{N}$ ' \\ Je crois que seul le temps te fera oublier cette image.}

Étant donné que des phrases comme (18) sont caractéristiques du français formel, Lamiroy \& De Mulder (2011: 309) affirment même que l'emploi des clivées en il n'y a que telles que (17) peut être « quasi-obligatoire » dans certains contextes en français parlé.

Pour conclure, le choix du format clivé plutôt que l'ordre des mots SV dans les exemples présentés ci-dessus s'explique au moins en partie par le souci de renforcer la cohérence textuelle, la préférence «syntagme court > syntagme long » et l'influence du registre (formel ou informel).

\section{La négation emphatique}

Dans cette section, je présenterai des clivées en il y a dont l'élément clivé est une expression négative telle que rien ou personne et qui peuvent être considérées comme des instances d'une «négation emphatique » par rapport à leurs équivalents canoniques à l'ordre des mots SV.

L'idée que certaines tournures ou expressions expriment une négation plus 'forte' par rapport à d'autres manières d'encoder la négation n'est pas nouvelle. Ainsi, Brunot (1922) mentionne les répétitions (non, non et non), l'ajout de certains adverbes (sûrement pas) et les jurons (diantre non!) comme des exemples de la négation emphatique (voir aussi Barnicaud et al., 1967 ; Floricic \& Mignon, 2007). Quirk et al. (1985: 1407) notent également que les phrases en there is peuvent être utilisées « as a means of emphasizing a negative », comme en (19).

\section{There's nothing I can do about it. (Quirk et al., 1985 : 1407)}

Quant au français, dans leur analyse des phrases existentielles en il y a et en there is telles que (20), Cappelle et al. (2016) affirment que la combinaison d'une expression existentielle (il $y$ a, there is), de la négation et d'un SN qui exprime une quantité minimale (un mot, l'ombre d'un doute) peut créer un effet emphatique. Leur idée est que si l'on nie l'existence d'une quantité minimale, on renforce l'absence de toute quantité plus importante («the denial of a minimal degree reinforces the absence of anything higher $»)$.

a. Y avait pas un mot de vrai dans son histoire.

b. Il n'y a pas l'ombre d'un doute.

(Cappelle et al., 2016) 
Cette analyse s'applique également aux clivées en il y a comme celles en (21) et en (22). Les SN clivés (pas) une seule personne et (pas) un renvoient tous les deux au nombre minimal de personnes imaginable et par conséquent, leur négation souligne le fait que personne n'a pas entendu parler de Dieu (c'est-à-dire que tout le monde a entendu parler de Dieu) en (21) et que personne n'a les couilles d'inviter Nicolas Sarkozy en (22).

$$
\begin{aligned}
& \text { - A : En quoi croient les tribus primitives? Dieu va t-il les détruire? } \\
& \text { - B : Tout peuple a reçu un messager pour le guider, il n'y a pas une seul } \\
& \text { personne qui n'a pas entendu parler de Dieu ou ses Prophètes (YCCQA) }
\end{aligned}
$$

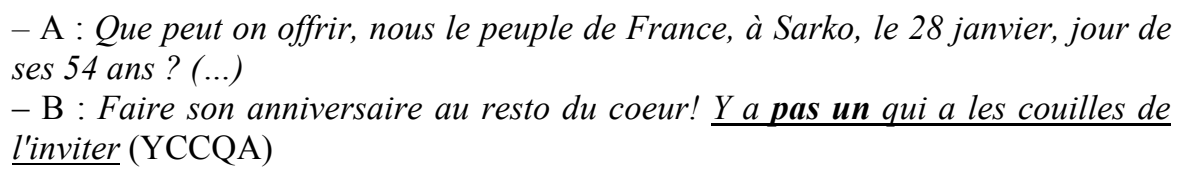

Il est grammaticalement possible d'exprimer les mêmes propositions selon l'ordre des mots $\mathrm{SV}$, comme en (23) et (24). Cependant, sans l'expression existentielle il y a, la négation de l'existence d' " une seule personne / un seul » est moins explicite.

(23) Pas une seule personne n'a pas entendu parler de Dieu ou ses Prophètes.

Pas un a les couilles de l'inviter.

Qui plus est, la phrase en (23) est plus difficile à comprendre en raison de la deuxième négation qui porte sur le syntagme verbal (n'a pas entendu). Dans ce sens, la clivée en (21) exemplifie la fonction de la désambiguation de la portée que Dufter (2009) attribue aux clivées en c'est : «By virtue of splitting up a single clause into two and singling out one major constituent, clefting is an excellent device to bring this constituent unambiguously under the scope of operators such as negation, hedges or the like » (Dufter, 2009: 108). ${ }^{\mathrm{V}} \mathrm{Je}$ reviendrai à la relation entre les clivées et la portée dans la section 6 .

\section{L'interprétation plurielle d'un indéfini singulier}

Dans ce qui suit, je montrerai que les clivées en il y a peuvent avoir la fonction d'indiquer qu'un SN indéfini singulier ne renvoie pas à une entité spécifique singulière, alors que dans l'équivalent selon l'ordre des mots SV, les SN indéfinis en position de sujet préverbal ont de préférence une interprétation spécifique, singulière. Autrement dit, le locuteur peut utiliser le format clivé pour rendre l'interprétation voulue plus claire.

Un premier exemple est présenté en (25). Cette clivée exprime un événement habituel, comme l'indique l'adverbe toujours.

\footnotetext{
" Dans ce milieu, poursuit-il, n'importe quel cadet qui brille un peu est, un jour ou l'autre, confronté au problème du dopage. A un moment ou à un autre, il y a toujours un type qui essaie de l'approcher. " (Le Monde)
}

Ce qui est important à noter est que l'élément clivé un type ne renvoie pas à un seul référent spécifique : ce n'est pas le même type qui approche le cadet à chaque reprise. Le quantifieur universel toujours a donc une portée large par rapport au quantifieur existentiel 
(toujours > il y a un type). Cependant, si le SN se trouve en tête de phrase, comme en (26), cette interprétation est moins évidente :

\section{À un moment ou à un autre, un type essaie toujours de l'approcher.}

Cette observation suit le «principe de la référence unique » de Kurtzman \& MacDonald (1993), selon lequel les SN indéfinis en position de sujet ou de topique sont de préférence interprétés comme des entités singulières. Je propose donc que l'une des fonctions du format clivé en (25) est de signaler plus explicitement que son équivalent canonique que le $\mathrm{SN}$ un type ne renvoie pas à un seul référent spécifique. C'est la structure syntaxique en 'phrase principale (il y a SN) + relative' qui permet de signaler cette lecture: premièrement, grâce à cette structure, le $\mathrm{SN}$ ne se trouve pas dans la position canonique des sujets préverbaux en tête de phrase, qui est associée à la lecture «singulière ». Deuxièmement, contrairement à l'équivalent canonique en (26), la structure de la clivée en (25) permet de faire précéder le SN un type par l'adverbe toujours. Or, cet ordre reflète la relation de portée entre ces deux expressions (toujours > un type). Il est possible que cette correspondance entre ordre des mots et relation de portée rende l'interprétation voulue plus évidente.

L'extrait en (27) présente un deuxième exemple. L'élément clivé un chameau n'a pas de référence spécifique singulière: ce n'est pas nécessairement le même chameau qui tombe chaque fois quand l'événement habituel se produit.

Le jeune réalisateur incarne aujourd'hui l'espoir d'un cinéma palestinien encore balbutiant. "Il tombe, le chameau. Le type le remet debout. Il tombe. C'est un chameau en bois, ou en paille, enfin, en cochonnerie, une horreur à fourguer aux touristes. Un petit chameau au milieu d'une longue file de chameaux du même métal. Mais eux ne tombent pas, sur l'étagère du magasin de souvenirs de Nazareth. Seulement, il y a toujours un chameau qui tombe, dans les meilleurs alignements, les dispositifs les plus sûrs. »(Le Monde)

De nouveau, l'interprétation non-singulière est indiquée plus clairement dans la clivée en (27) que dans la phrase à l'ordre des mots SV, où un chameau est un sujet préverbal. Et, comme en (25), l'ordre de l'adverbe (toujours) et du SN (un chameau) correspond à la portée large de l'adverbe par rapport au chameau (toujours $>$ il y a un chameau), contrairement à l'équivalent canonique en (28).

\section{Seulement, un chameau tombe toujours.}

Pour conclure, la structure biclausale des clivées en il y a permet de placer un SN indéfini singulier dans une position où ce $\mathrm{SN}$ n'est pas interprété comme renvoyant à une entité spécifique singulière. Cette interprétation est moins évidente dans les équivalents canoniques de ces clivées, où le $\mathrm{SN}$ se trouve en position de sujet préverbal, précédent le quantifieur universel toujours.

\section{Conclusion}

Le but de cet article était de montrer que les clivées en il y a sont plus que de simples marqueurs de focus et que, par conséquent, la structure informationnelle ne permet pas à elle seule d'expliquer pourquoi le locuteur opte pour le format clivé plutôt que pour son équivalent canonique selon l'ordre des mots SV. Pour ce faire, j'ai présenté des exemples de corpus de clivées en il y a ayant des fonctions qui ne sont pas directement liées aux 
notions de 'topique' et de 'focus'. Autrement dit, le format clivé peut être exploité pour des raisons diverses plutôt que pour le seul but d'introduire un SN focal et non-topical : il y a des fonctions supplémentaires qui viennent s'ajouter à la fonction de marquer une certaine structure informationnelle.

Les fonctions que j'ai tenté d'illustrer et d'expliquer sont les suivantes. Primo, les clivées en il y a à élément clivée anaphorique permettent de renforcer le lien entre le contexte précédent et la prédication dans la relative. Ce lien est moins évident dans l'équivalent à l'ordre des mots SV. Secundo, quand les clivées en il y a contiennent une négation et un SN exprimant une quantité minimale, la clivée peut être considérée comme une instance de la négation emphatique. Tertio, l'emploi du format clivé peut signaler qu'un SN indéfini singulier est à interpréter de manière non-spécifique, non-singulier. Cette interprétation est moins claire dans l'équivalent canonique selon l'ordre des mots SV.

Pour conclure, en analysant des clivées en il y $a$ authentiques dans leur contexte d'apparition, j'ai pu montrer que, contrairement à ce que laissent penser certaines études antérieures, la motivation de ces clivées ne se réduit pas à la focalisation. Dans ce sens, cette contribution rejoint différentes analyses des clivées en c'est, qui, elles aussi, ont des fonctions diverses. Tout en reconnaissant que la structure informationnelle joue un rôle important pour expliquer pourquoi les locuteurs utilisent les clivées, je tiens donc à souligner que, comme c'est souvent le cas, c'est une multitude de facteurs qui déterminent le choix d'une structure (une clivée) au lieu d'une autre (phrase canonique) plutôt qu'un seul facteur.

\section{Références bibliographiques}

Ashby, W. J. (1995). French presentational structures. Dans J. Amastae, G. Goodall, M. Montalbetti \& M. Phinney (éds.), Contemporary research in Romance linguistics (pp. 91-104). Amsterdam/Philadelphia: John Benjamins.

Ashby, W. J. (1999). Au sujet de quoi? La fonction du sujet grammatical, du complément d'objet direct, et de la construction présentative en français parlé. The French Review, 72(3), 481-492.

Barnicaud, G., Compare, M. A., Ducrot, O. \& Duval, A. (1967). Le problème de la négation dans diverses grammaires françaises. Langages, 2(7), 58-73.

Behagel, O. (1909). Beziehungen zwischen Umfang und Reihenfolge von Satzgliedern. Indogermanische Forschungen, 25, 110-142.

Blanche-Benveniste, C. (1997). Approches de la langue parlée en français. Paris: Ophrys.

Blanche-Benveniste, C. (2006). Les clivées françaises de type: C'est comme ça que, c'est pour ça que, cest là que tout a commencé. Moderna Språk, 100(2), 273-287.

Brunot, F. (1922). La pensée et la langue: Méthode, principes et plan d'une théorie nouvelle du langage appliquée au français. Paris: Masson.

Cappeau, P. \& Deulofeu, J. (2001). Partition et topicalisation: Il y en a "stabilisateur" de sujets et de topiques indéfinis. Cahiers de praxématique, 37, 45-82.

Cappelle, B., Carlier, A., Fagard, B. \& Meulleman, M. (2016). Negative existentials - Distinct syntactic, semantic and pragmatic properties, Paper presented at Societas Linguistica Europaea [SLE] 49. Naples.

Charolles, M. \& Vigier, D. (2005). Les adverbiaux en position préverbale: Portée cadrative et organisation du discours. Langue française, 148(4), 9-30.

Choi-Jonin, I. \& Lagae, V. (2005). Il y a des gens ils ont mauvais caractère. A propos du rôle de il y $a$. Dans A. Murguía (éd.), Sens et références. Mélanges Georges Kleiber (pp. 39-66). Tübingen: Gunter Narr.

Clech-Darbon, A., Rebuschi, G. \& Rialland, A. (1999). Are there cleft sentences in French? Dans L. Tuller \& G. Rebuschi (éds.), The grammar of focus (pp. 83-118). Amsterdam: John Benjamins.

Delin, J. (1989). Cleft constructions in discourse. Edinburgh: University of Edinburgh PhD dissertation.

Delin, J. \& Oberlander, J. (1995). Syntactic constraints on discourse structure: The case of $i t$-clefts. Linguistics, 33(3), 465-500. 
Destruel, E. (2013). The French c'est-cleft: Empirical studies of its meaning and use. Austin: University of Texas at Austin $\mathrm{PhD}$ dissertation.

Doetjes, J., Rebuschi, G. \& Rialland, A. (2004). Cleft Sentences. Dans F. Corblin \& H. De Swart (éds.), Handbook of French semantics (pp. 529-552). Stanford: CSLI Publications.

Dufter, A. (2008). On explaining the rise of c'est-clefts in French. Dans U. Detges \& R. Waltereit (éds.), The paradox of grammatical change: Perspectives from Romance (pp. 31-56). Amsterdam: John Benjamins.

Dufter, A. (2009). Clefting and Discourse organization: Comparing Germanic and Romance. Dans A. Dufter \& D. Jacob (éds.), Focus and Background in Romance languages (pp. 83-121). Amsterdam/Philadelphia: John Benjamins.

Floricic, F. \& Mignon, F. (2007). Négation et réduplication intensive en français et en italien. Dans F. Floricic (éd.), La négation dans les langues romanes (pp. 117-136). Amsterdam: John Benjamins.

Giry-Schneider, J. (1988). L'interprétation événementielle des phrases en il y a. Lingvisticae Investigationes, 12(1), 85-100.

Givón, T. (1983). Topic continuity in discourse: An introduction. Dans T. Givón (éd.), Topic contituity in discourse (pp. 1-42). Amsterdam/Philadelphia: John Benjamins.

Hawkins, J. A. (1994). A performance theory of order and constituency. Cambridge: Cambridge University Press.

Jacob, D. (2015). Anaphorische Spaltsätze im Französischen: Grammatik - Text - Rhetorik. Dans S. Adam, D. Jacob \& M. Schecker (éds.), Informationsstrukturen in Kontrast: Strukturen, Kompositionen und Strategien (pp. 101-122). Frankfurt am Main: Peter Lang.

Jeanjean, C. (1979). Soit y avait le poisson soit y avait ce rôti farci: Étude de la construction il y a dans la syntaxe du français. Recherches sur le Français Parlé, 2, 121-162.

Jullien, S. (2007). Prosodic, syntactic and semantico-pragmatic parameters as clues for projection: The case of "il y a". Nouveaux cahiers de linguistique française, 28, 279-297.

Karssenberg, L. (2016a). French il y a clefts, existential sentences and the focus-marking hypothesis. Journal of French Language Studies 27 (3), 405-430.

Karssenberg, L. (2016b). Il n'y a que Superman qui porte le slip par-dessus le pantalon: Les clivées en il n'y a que x qui. Dans 5e Congrès Mondial de Linguistique Française Vol. 27 (pp. 1-14). Les Ulis: EDP Sciences.

Karssenberg, L. (2017a). Specificerende il y a-clefts (er is-clefts). Handelingen der Koninklijke ZuidNederlandse Maatschappij voor Taal- en Letterkunde en Geschiedenis (KZM), 77-91.

Karssenberg, L. (2017b). Ya les oiseaux qui chantent. A corpus analysis of French il y a clefts. KU Leuven $\mathrm{PhD}$ dissertation.

Karssenberg, L. \& Lahousse, K. (à venir). The information structure of French il y a clefts \& c'est clefts: A corpus-based analysis. Linguistics.

Katz, S. (2000). Categories of c'est-cleft constructions. Canadian Journal of Linguistics/Revue canadienne de linguistique, 45(2), 253-273.

Krötsch, M. \& Sabban, A. (1990). "Bleu, je veux" - Remarques sur la focalisation en français. Zeitschrift für romanische Philologie, 106(1), 80-98.

Kurtzman, H. S. \& MacDonald, M. C. (1993). Resolution of quantifier scope ambiguities. Cognition, 48(3), 243-279.

Lagae, V. \& Rouget, C. (1998). Quelques réflexions sur les relatives prédicatives. Dans M. Bilger, K. Van den Eynde \& F. Gadet (éds.), Analyse linguistique et approches de l'oral. Recueil d'études offert en hommage à Claire Blanche-Benveniste (pp. 313-325). Leuven/Paris: Peeters.

Lahousse, K. \& Borremans, M. (2014). The distribution of functional-pragmatic types of clefts in adverbial clauses. Linguistics, 52(3), 793-836.

Lahousse, K. \& Lamiroy, B. (2015). C'est ainsi que: Grammaticalisation ou lexicalisation ou les deux à la fois ? Journal of French Language Studies, 1-25.

Lambrecht, K. (1986). Pragmatically motivated syntax. Presentational cleft constructions in spoken French. Dans 22nd Conference of the Chicago Linguistic Society. Papers from the parasession on Pragmatics and Grammatical Theory (pp. 115-126). Chicago: Chicago Linguistic Society.

Lambrecht, K. (1988). Presentational cleft constructions in spoken French. Dans J. Haiman \& S.A. Thompson (éds.), Clause combining in grammar and discourse (pp. 135-179). Amsterdam/Philadelphia: John Benjamins.

Lambrecht, K. (1994). Information structure and sentence form: Topic, focus and the mental representations of discourse referents. Cambridge: Cambridge University Press. 
Lambrecht, K. (2000a). Prédication seconde et structure informationnelle: La relative de perception comme construction présentative. Langue française, 127(1), 49-66.

Lambrecht, K. (2000b). When subjects behave like objects: A markedness analysis of sentence-focus constructions across languages. Studies in Language, 24(3), 611-682.

Lambrecht, K. (2001). A framework for the analysis of cleft constructions. Linguistics, 39(3), 463516.

Lambrecht, K. (2002). Topic, focus and secondary predication. The French presentational relative construction. Dans C. Beyssade, R. Bok-Bennema, F. Drijkoningen \& P. Monachesi (éds.), Romance Languages and Linguistic Theory 2000 (pp. 171-212). Amsterdam/Philadelphia: John Benjamins.

Lamiroy, B. \& Charolles, M. (2004). Simplement, seulement, malheureusement, heureusement. Travaux de linguistique, 49(2), 57-79.

Lamiroy, B. \& De Mulder, W. (2011). Degrees of grammaticalization across languages. Dans B. Heine \& H. Narrog (éds.), The Oxford Handbook of Grammaticalization (pp. 302-317). Oxford: Oxford University Press.

Léard, J.-M. (1992). Les gallicismes. Étude syntaxique et sémantique. Paris/Leuven: Duculot.

Matić, D. \& Wedgwood, D. (2012). The meanings of focus: The significance of an interpretationbased category in cross-linguistic analysis. Journal of Linguistics, 49(1), 127-163.

Mertens, P. (2008). Syntaxe, prosodie et structure informationnelle: Une approche prédictive pour l'analyse de l'intonation dans le discours. Travaux de linguistique, 56(1), 87-124.

Mertens, P. (2012). La prosodie des clivées. Dans S. Caddéo, M.-N. Roubaud, M. Rouquier \& F. Sabio (éds.), Penser les langues avec Claire Blanche-Benveniste (pp. 127-139). Aix-enProvence: Presses Universitaires de Provence.

Molinier, C. \& Levrier, F. (2000). Grammaire des adverbes: Description des formes en -ment. Geneva: Librairie Droz.

Pekarek Doehler, S. (2015). Grammar, projection and turn-organization: (il) y a NP 'there is NP' as a projector construction in French talk-in-interaction. Dans A. Deppermann \& S. Günthner (éds.), Temporality in interaction (pp. 173-199). Amsterdam: John Benjamins.

Pitavy, C. (2009). "Y a-t-il un chien qui aboie ... dans les didascalies ?" Theatrical discourse and enunciation. Review of Interdisciplinary Centre for Studies of Contemporary Discursive Forms(4), 123-146.

Quirk, R., Greenbaum, S., Leech, G. \& Svartvik, J. (1985). A comprehensive grammar of the English language. London: Longman.

Rialland, A., Rebuschi, G. \& Doetjes, J. (2002). What is focused in C'est XP qui/que cleft sentences in French? Dans B. Bel \& I. Marlien (éds.), Speech Prosody (pp. 595-598). Aix-en-Provence: ISCA Archive.

Secova, M. (2010). Discourse-pragmatic features of spoken French: Analysis and pedagogical implications. London: Queen Mary, University of London PhD dissertation.

Smits, R. J. C. (1989). Eurogrammar. The relative and cleft constructions in the Germanic and Romance languages. Dordrecht: Foris Publications.

Van De Velde, D. (1995). Le spectre nominal: Des noms de matières aux noms d'abstractions. Leuven: Peeters.

Verlinde, S. \& Selva, T. (2001a). Corpus-based versus intuition-based lexicography: Defining a word list for a French learners' dictionary. Dans Corpus Linguistics 2001 (pp. 594-598). Lancaster: http://ucrel.lancs.ac.uk/publications/cl2003/CL2001\%20conference/.

Verlinde, S. \& Selva, T. (2001b). Nomenclature de dictionnaire et analyse de corpus. Cahiers de Lexicologie, 79(2), 113-139.

Verwimp, L. \& Lahousse, K. (2016). Definite il y a-clefts in spoken French. Journal of French Language Studies, 1-28.

Wasow, T. (2002). Postverbal behavior. Stanford, CA: CSLI Publications.

Willems, D. \& Meulleman, M. (2010). "Il y des gens ils viennent acheter des aspirines pour faire de l'eau gazeuse". Sur les raisons d'être des structures parataxiques en il y $a$. Dans M.-J. Béguelin, M. Avanzi \& G. Corminboeuf (éds.), La parataxe. Tome 2: structures, marquages et exploitations discursives (pp. 167-184). Bern: Peter Lang. 
${ }^{\mathrm{i}}$ Selon plusieurs auteurs, les phrases en il y a... qui ont la fonction d'éviter que des SN indéfinis apparaissent en tête de phrase (Jeanjean, 1979 ; Léard, 1992 : section 3.3.2 ; Van De Velde, 1995 : 28 ; Blanche-Benveniste, 1997 ; Cappeau \& Deulofeu, 2001). Cependant, ces auteurs ne considèrent pas cette structure comme un type de clivée. Voir Karssenberg (2017b : 206-209) sur le lien entre cette hypothèse et les fonctions informationnelles des clivées en il y $a$.

${ }^{\text {ii }}$ Le corpus $Y C C Q A$ a été créé et est géré par Hendrik de Smet (KU Leuven).

iii Voir aussi la notion de « topic continuity 》 (Givón, 1983).

${ }^{\text {iv }}$ Voir aussi Charolles \& Vigier (2005) sur les différences d'interprétation des adverbes en position préverbale ou postverbale.

${ }^{\vee}$ Dufter (2009 : 108) présente (i) pour illustrer la fonction des clivées en c'est liée à la portée :

(i) Ce n'est pas l'Europe qui s'est élargie, mais bien l'Union européenne. 